\title{
Optic Neuritis Possibly Induced by Anti-PD-L1 Antibody Treatment in a Patient with Non-Small Cell Lung Carcinoma
}

\author{
Sotaro Mori Takuji Kurimoto Kaori Ueda Hiroko Enomoto \\ Mari Sakamoto Yukako Keshi Yuko Yamada Makoto Nakamura \\ Division of Ophthalmology, Department of Surgery, Kobe University Graduate School of \\ Medicine, Kobe, Japan
}

\section{Keywords}

Optic neuritis · Anti-PD-L1 antibody · Immune-related adverse effects · Hypopituitarism

\begin{abstract}
Background: Recent immune therapy with checkpoint inhibitors (CPIs) has demonstrated remarkable antitumor effects on specific tumors, such as malignant lymphoma and non-small cell lung carcinoma. By contrast, CPIs cause an imbalance in the immune system, triggering a wide range of immunological side effects termed immune-related adverse effects (irAEs). Here, we report a rare case of optic neuritis and hypopituitarism during anti-programmed deathligand 1 (PD-L1) antibody treatment. Case Presentation: A patient with non-small cell lung carcinoma received anti-PD-L1 antibody treatment every 3 weeks; however, the patient started experiencing headaches, general fatigue, anorexia, and diarrhea approximately 1 year after the initiation of the treatment. Moreover, sudden visual loss of the right eye occurred 1 week after the interruption of the anti-PD-L1 antibody treatment. MRI findings showed gadolinium enhancement in the left optic nerve, but no enlargement of the pituitary gland and stalk. Laboratory data showed decreased serum adrenocorticotropic hormone (ACTH), cortisol, and free $\mathrm{T}_{4}$ levels, and a hormone tolerance test indicated hypopituitarism, hypothyroidism, and hypoadrenocorticism. The central scotoma caused by optic neuritis completely disappeared immediately after a course of steroid pulse therapy, and no recurrence occurred up to 2 years
\end{abstract}


after initiation of the steroid pulse therapy while replacement therapy for hypothyroidism and hypoadrenocorticism was continued. Conclusions: The patient presented with optic neuritis and hypopituitarism, possibly due to irAEs of the anti-PD-L1 antibody treatment. Steroid pulse therapy was effective for optic neuritis, suggesting underlying immunological mechanisms. Neurological complications including optic neuritis should be considered when examining patients with cancer undergoing CPI treatment.

\section{Background}

Checkpoint inhibitors (CPIs) have been established as a promising alternative treatment for various cancers, including melanoma, lung cancer, mesothelioma, renal cancer, lymphoma, and metastatic prostate cancer. Among CPIs, anti-cytotoxic T-lymphocyte-associated-4 (CTLA-4) antibodies were the first to be approved as an antitumor drug for melanoma, followed by the anti-programmed death 1 (PD-1) and anti-programmed death-ligand 1 (PD-L1) antibodies. PD-1 is expressed on a broader range of immune cells including CD4+ and CD8+ T cells, B cells, natural killer cells, and T-regulatory cells. There are two ligands for PD-1: PD-L1 and PD-L2. PD-L1 is expressed on T and B cells, dendritic cells, and macrophages. Upon induction by various cytokines, PD-L1 activates PD-1 on T cells and inhibits T-cell proliferation and production of proinflammatory Th1 cytokines, including IFN- $\gamma$ and IL-2. Therefore, by this cascade of reactions, the PD-1/PD-L1 pathway plays a critical role in the control of T-cell activity in inflamed tissues. Similarly, tumor cells can exploit the PD-1/PD-L1 pathway to escape host T-cell-regulated immune surveillance. Antibodies against PD-1, such as nivolumab, pembrolizumab, and pidilizumab, or those against PD-L1, such as MEDI4736 and MPDL3280A, prevent T-cell inactivation by blocking the PD-1/PD-L1 pathway between host T cells and tumor cells, which eventually increase the antitumor T-cell response. Anti-PD-1/PD-L1 antibodies have been proven to induce marked cytoreductive effects against non-small cell lung carcinoma $[1,2]$. However, by causing immune system imbalance, these new immunotherapies also generate dysimmune toxicities termed immune-related adverse effects (irAEs) that mainly involve the gut, skin, liver, endocrine gland, lung, and nervous system, and thus, can affect any organ [3]. IrAEs reportedly occur in up to $90 \%$ of patients treated with an antiCTLA-4 antibody and in 70\% of patients treated with an anti-PD-1/PD-L1 antibody. Among patients experiencing irAEs, $1-5 \%$ show neurological dysfunction and $<1 \%$ have ophthalmic complications $[4,5]$. Optic neuritis has been recently reported in association with anti-CTLA4 antibody treatment [6-8]; however, a detailed description of optic neuritis associated with anti-PD-1 or anti-PD-L1 antibody treatment has not yet been published [9]. Here, we report the rare case of a patient with optic neuritis as an irAE, which occurred 1 year after the initiation of anti-PD-L1 antibody (MPDL3280A) treatment, and was accompanied by hypopituitarism due to the decreased release of adrenocorticotropic hormone (ACTH) and thyroid-stimulating hormone (TSH).

\section{Case Presentation}

A 64-year-old man was diagnosed 2 years previously with stage IV non-small cell lung carcinoma and received four cycles of cisplatin and Abraxane combination therapy as the firstline treatment and two cycles of pemetrexed as the second-line treatment in the Department of Thoracic Oncology, Hyogo Cancer Center. However, the treatment failed to show tumor 


\section{Case Reports in Ophthalmology}

regression. Stereotaxic radiation therapy successfully retarded the metastatic lesions in the hypothalamus and in areas adjacent to the lateral ventricle.

The patient subsequently participated in a phase III clinical trial of anti-PD-L1 antibody (MPDL-3280A) treatment, which dramatically reduced the size and number of lung tumors at the 1-year follow-up. However, the patient also suffered from general fatigue, anorexia, diarrhea, and bilateral upper limb pain. As a result, the anti-PD-L1 antibody treatment was discontinued. Gastrointestinal endoscopy revealed reflux esophagitis and erosive duodenal inflammation, which were treated with proton pump inhibitors. After the withdrawal of antiPD-L1 antibody treatment, his diarrhea spontaneously disappeared and the cause of the bilateral upper limb pain remained unclear even after electrical electromyography and muscle biopsy.

Approximately 12 months after the initiation of the anti-PD-L1 antibody treatment, the patient experienced sudden visual loss in the left eye without eye movement pain and was referred to our hospital. At the initial inspection, the left eye showed a best-corrected visual acuity (BCVA) of 0.01, a nasal visual field defect, and an enlarged blind spot; moreover, the right eye had no visual field defect (Fig. 1a, b). The anterior segment and media were normal. The direct reaction of light reflex in the left eye was weakened, and a relative afferent pupillary defect was detected. The left optic disk was swollen and showed mild venous congestion without bleeding; it exhibited dye leakage on fluorescein angiography. There were no abnormalities of choroidal circulation, retinal vessels, or parenchyma (Fig. 2a-C). Brain magnetic resonance imaging (MRI) with gadolinium enhancement showed a high-intensity lesion in the left optic nerve, but no abnormalities in the pituitary body and stalk (Fig. 3a-c). Anti-aquaporin-4 antibodies were absent, and herpes simplex virus and varicella-zoster virus antibody titers were not elevated. The cerebral spinal fluid contained elevated total protein $(69 \mathrm{mg} / \mathrm{dL})$ and myelin basic protein ( $105 \mathrm{ng} / \mathrm{mL}$; normal range, $>102 \mathrm{ng} / \mathrm{mL}$ ) levels, but lacked cells and oligoclonal IgG bands with normal IgG index, discriminating infectious and demyelinating diseases. ACTH, free $\mathrm{T}_{4}$, and cortisol levels were $4.0 \mathrm{pg} / \mathrm{mL}, 0.64 \mathrm{ng} / \mathrm{mL}$, and $<0.2 \mu \mathrm{g} / \mathrm{dL}$, respectively, all below the normal range. TSH $(1.54 \mu \mathrm{IU} / \mathrm{mL})$ and growth hormone $(0.38 \mathrm{ng} / \mathrm{mL})$ were within the normal range, whereas both follicle-stimulating hormone and prolactin were elevated at $9.4 \mathrm{mIU} / \mathrm{mL}$ (normal range, $2-8.3$ ) and $17.8 \mathrm{ng} / \mathrm{mL}$ (normal range, 3.58-12.78), respectively. Furthermore, the combined triple-hormone (insulin, thyrotropin-releasing hormone, and luteinizing hormone-releasing hormone) tolerance test revealed unresponsive ACTH release and decreased TSH responsiveness, although growth hormone, luteinizing hormone, follicle-stimulating hormone, and prolactin were responsive. Based on these clinical findings, the present patient was diagnosed with left optic neuritis accompanied by hypopituitarism, hypoadrenocortism, and hypothyroidism, probably induced by the PD-L1 treatment.

Steroid pulse therapy with intravenous $1.0 \mathrm{~g}$ methylprednisolone for 3 days followed by $30 \mathrm{mg}$ PO prednisolone administration was conducted. Replacement therapy with $25 \mu \mathrm{g}$ levothyroxine and $20 \mathrm{mg}$ p.o. hydrocortisone was included to treat the hypothyroidism and hypoadrenocortism. After the steroid pulse and replacement therapies, the visual field markedly recovered and optic disk swelling subsided (Fig. 1c). The posttreatment orbital MRI showed decreased gadolinium enhancement in the left optic nerve (Fig. 3d). BCVA in the left eye returned to 0.2 , equivalent to the value prior to disease onset, which was low due to amblyopia since childhood. These treatment regimens also diminished the general symptoms related to hypopituitarism and normalized the serum ACTH, cortisol, and free $\mathrm{T}_{4}$ levels. No recurrence of optic neuritis occurred at the final follow-up 2 years after the treatment. 


\section{Case Reports in Ophthalmology}

\section{Discussion}

The multicenter clinical trials on CPIs have revealed not only significant tumor-reductive effects but also a high occurrence rate of irAEs. A variety of ocular complications, such as peripheral ulcerative keratitis, uveitis, Vogt-Koyanagi-Harada disease, inflammatory orbital disease, choroid neovascularization, melanoma-associated retinopathy, and polymyalgia rheumatoid giant cell arteritis, have been previously reported, predominately in cases treated with anti-CTLA- 4 antibodies. Of these, optic neuritis is a rare complication, with only 2 cases reported to be caused by anti-CTLA- 4 and 1 case caused by anti-PD-L1 antibody treatment $[6,8$, 9]. Wilson et al. [6] reported a case of metastatic melanoma accompanied by bilateral optic neuritis and hypophysitis, in which the patient became blind in the right eye despite combination therapy with steroid pulse, intravenous immunoglobulin, and plasma exchanges. Such resistance to treatments implied underlying vascular pathological events rather than immunological events. Yeh and Francis [7] also reported a case of sudden visual loss with cells in the anterior chamber, posterior synechiae, and an optic disk edema in both eyes during antiCTLA-4 antibody treatment. Topical prednisolone reduced the inflammatory changes and improved visual acuity. Although the inflammatory change of the optic nerve was confirmed with enhanced MRI, this case was presumed to be papillitis induced by the anti-CTLA- 4 antibody application. The third case was treated with nivolumab, an anti-PD-1 antibody, in combination with a peptide vaccine, and was complicated with optic neuritis. However, a detailed description of the clinical course was not documented. To our knowledge, our patient is the first to present with optic neuritis accompanied by hypopituitarism and hypoadrenocorticism related to anti-PD-L1 antibody treatment.

Approximately 5-10\% of patients receiving CPIs are likely to develop endocrine irAEs of any grade that damage the thyroid or pituitary glands $[10,11]$. Hypophysitis is observed in up to $10 \%$ of patients receiving anti-CTLA- 4 antibody treatment; however, the incidence in patients undergoing anti-PD-1/PD-L1 antibody treatment is much lower $(<0.1 \%)[3,12]$. Ryder et al. [11] reported that the mean time to the onset of hypophysitis symptoms following initiation of anti-CTLA-4 antibody treatment was 4 months among 19 patients complicated with hypophysitis; of these, 2 patients had delayed onset at 8 and 19 months [11]. In studies regarding anti-PD-1 antibody treatment, the mean time to onset of hypophysitis related to nivolumab and pembrolizumab was 4.9 months (range, 1.4-11) and 3.3 months (range, 1 day to 11.9 months), respectively $[13,14]$. Recently, two case reports demonstrated late-onset adrenal insufficiency associated with hypophysitis following PD-L1 antibody treatment. The onset times of hypophysitis in each case were 56 and 52 weeks after the initiation of therapy [15]. Although the exact mechanisms underlying the delayed onset of hypophysitis induced by CPIs remain unclear, the onset time of hypophysitis in our case seems similar to these reports.

Symptoms of hypophysitis vary and include headache, mild fatigue, arthralgias, behavioral change, and loss of libido; the nonspecific nature of these symptoms can make hypophysitis difficult to diagnose. The most common neuroimaging finding in hypophysitis is a slight or mild enlargement of the pituitary gland with variable enhancement. However, pituitary structural changes can be missed because pituitary enlargement is transient and often mild in patients with hypophysitis induced by CPIs. This is particularly true if imaging is not performed immediately after the diagnosis of hypophysitis. Some authors have documented cases of hypophysitis wherein pituitary enlargement resolved after 7-12 days of steroid pulse therapy and other reports have documented cases of CTLA-4-induced hypophysitis with nor- 
mal MRI appearances [16]. Faje et al. [17] investigated the clinical characteristics of hypophysitis and endocrine dysfunction induced by anti-CTLA- 4 antibody treatment. The authors emphasized that neuroimaging abnormalities preceded symptoms in approximately $50 \%$ of patients. Our patient showed low serum ACTH, free $\mathrm{T}_{4}$, and cortisol levels and dysfunctional $\mathrm{ACTH}$, free $\mathrm{T}_{4}$, and TSH release in the hormone tolerance test, supporting the presence of hypophysitis causing hypopituitarism, which could not be detected by MRI because of the delay in image acquisition.

The National Cancer Institution Common Terminology Criteria Adverse Events (NCI CTCAE) severity grade version 4 stipulates the overall management approach and actions for irAEs associated with CPIs. According to the CTCAE, oral steroid application with an initial dose of $1-2 \mathrm{mg} / \mathrm{kg}$ is recommended for grade 2 irAEs. If the severity is more than grade 3 , CPIs should be discontinued transiently or permanently in addition to steroid pulse therapy [18]. The optic neuritis of our patient corresponded with grade 4 severity: blindness with less than 20/200 in the affected eye, which is an indication for immediate steroid treatment. Consequently, the patient was sensitive to steroid pulse therapy without tumor progression for at least 2 years.

In conclusion, we reported a case presenting with optic neuritis as an irAE, which is possibly associated with anti-PD-L1 antibody treatment. Ophthalmologists and neurologists should consider the possibility of optic neuritis as an irAE when examining cancer patients, and it is desirable to perform steroid pulse therapy promptly and appropriately.

\section{Acknowledgments}

We are grateful to Enago (www.enago.jp) for the English language review.

\section{Statement of Ethics}

This report was published with the permission and informed consent of the patient.

\section{Disclosure Statement}

None of the authors has any conflicts of interest.

\section{References}

1 Brahmer JR, Tykodi SS, Chow LQ, Hwu WJ, Topalian SL, Hwu P, et al. Safety and activity of anti-PD-L1 antibody in patients with advanced cancer. N Engl J Med. 2012 Jun;366(26):2455-65.

2 Gandini S, Massi D, Mandalà M. PD-L1 expression in cancer patients receiving anti PD-1/PD-L1 antibodies: A systematic review and meta-analysis. Crit Rev Oncol Hematol. 2016 Apr;100:88-98.

3 Michot JM, Bigenwald C, Champiat S, Collins M, Carbonnel F, Postel-Vinay S, et al. Immune-related adverse events with immune checkpoint blockade: a comprehensive review. Eur J Cancer. 2016 Feb;54:139-48.

4 Della Vittoria Scarpati G, Fusciello C, Perri F, Sabbatino F, Ferrone S, Carlomagno C, et al. Ipilimumab in the treatment of metastatic melanoma: management of adverse events. OncoTargets Ther. 2014 Feb;7:203-9.

5 Antoun J, Titah C, Cochereau I. Ocular and orbital side-effects of checkpoint inhibitors: a review article. Curr Opin Oncol. 2016 Jul;28(4):288-94

6 Wilson MA, Guld K, Galetta S, Walsh RD, Kharlip J, Tamhankar M, et al. Acute visual loss after ipilimumab treatment for metastatic melanoma. J Immunother Cancer. 2016 Oct;4(1):66. 
7 Sheldon CA, Kharlip J, Tamhankar MA. Inflammatory Orbitopathy Associated With Ipilimumab. Ophthal Plast Reconstr Surg. 2017 May/Jun;33(3S Suppl 1):S155-8.

8 Yeh OL, Francis CE. Ipilimumab-associated bilateral optic neuropathy. J Neuroophthalmol. 2015 Jun;35(2):144-7.

9 Weber JS, Kudchadkar RR, Yu B, Gallenstein D, Horak CE, Inzunza HD, et al. Safety, efficacy, and biomarkers of nivolumab with vaccine in ipilimumab-refractory or -naive melanoma. J Clin Oncol. 2013 Dec;31(34):4311-8.

10 Topalian SL, Hodi FS, Brahmer JR, Gettinger SN, Smith DC, McDermott DF, et al. Safety, activity, and immune correlates of anti-PD-1 antibody in cancer. N Engl J Med. 2012 Jun;366(26):2443-54.

11 Ryder M, Callahan M, Postow MA, Wolchok J, Fagin JA. Endocrine-related adverse events following ipilimumab in patients with advanced melanoma: a comprehensive retrospective review from a single institution. Endocr Relat Cancer. 2014 Mar;21(2):371-81.

12 Faje A. Hypophysitis: evaluation and Management. Clin Diabetes Endocrinol. 2016 Sep;2(1):15.

13 OPDIVO® (nivolumab) Prescribing Information (revised 4/2018). https://packageinserts.bms.com/pi/pi_opdivo.pdf (accessed Jun 10, 2018)

14 KEYTRUDA® (pembrolizumab) Prescribing Information (revised 11/2017). https://www.merk.com/product/usa/pi_circulars/k/keytruda/k.eytruda_pi.pdf (accessed Jun 10, 2018)

15 Kanie K, Iguchi G, Bando H, Fujita Y, Odake Y, Yoshida K, et al. Two Cases of Atezolizumab-Induced Hypophysitis. J Endocr Soc. 2017 Dec;2(1):91-5.

16 Bertrand A, Kostine M, Barnetche T, Truchetet ME, Schaeverbeke T. Immune related adverse events associated with anti-CTLA-4 antibodies: systematic review and meta-analysis. BMC Med. 2015 Sep;13(1):211.

17 Faje AT, Sullivan R, Lawrence D, Tritos NA, Fadden R, Klibanski A, et al. Ipilimumab-induced hypophysitis: a detailed longitudinal analysis in a large cohort of patients with metastatic melanoma. J Clin Endocrinol Metab. 2014 Nov;99(11):4078-85.

18 Kumar V, Chaudhary N, Garg M, Floudas CS, Soni P, Chandra AB. Current Diagnosis and Management of Immune Related Adverse Events (irAEs) Induced by Immune Checkpoint Inhibitor Therapy. Front Pharmacol. 2017 Feb;8:49. 


\section{Case Reports in \\ Ophthalmology}

Case Rep Ophthalmol 2018;9:348-356

DOI: $10.1159 / 000491075$

2018 The Author(s). Published by S. Karger AG, Basel www.karger.com/cop

Mori et al.: Optic Neuritis Possibly Induced by anti-PD-L1 Antibody Treatment
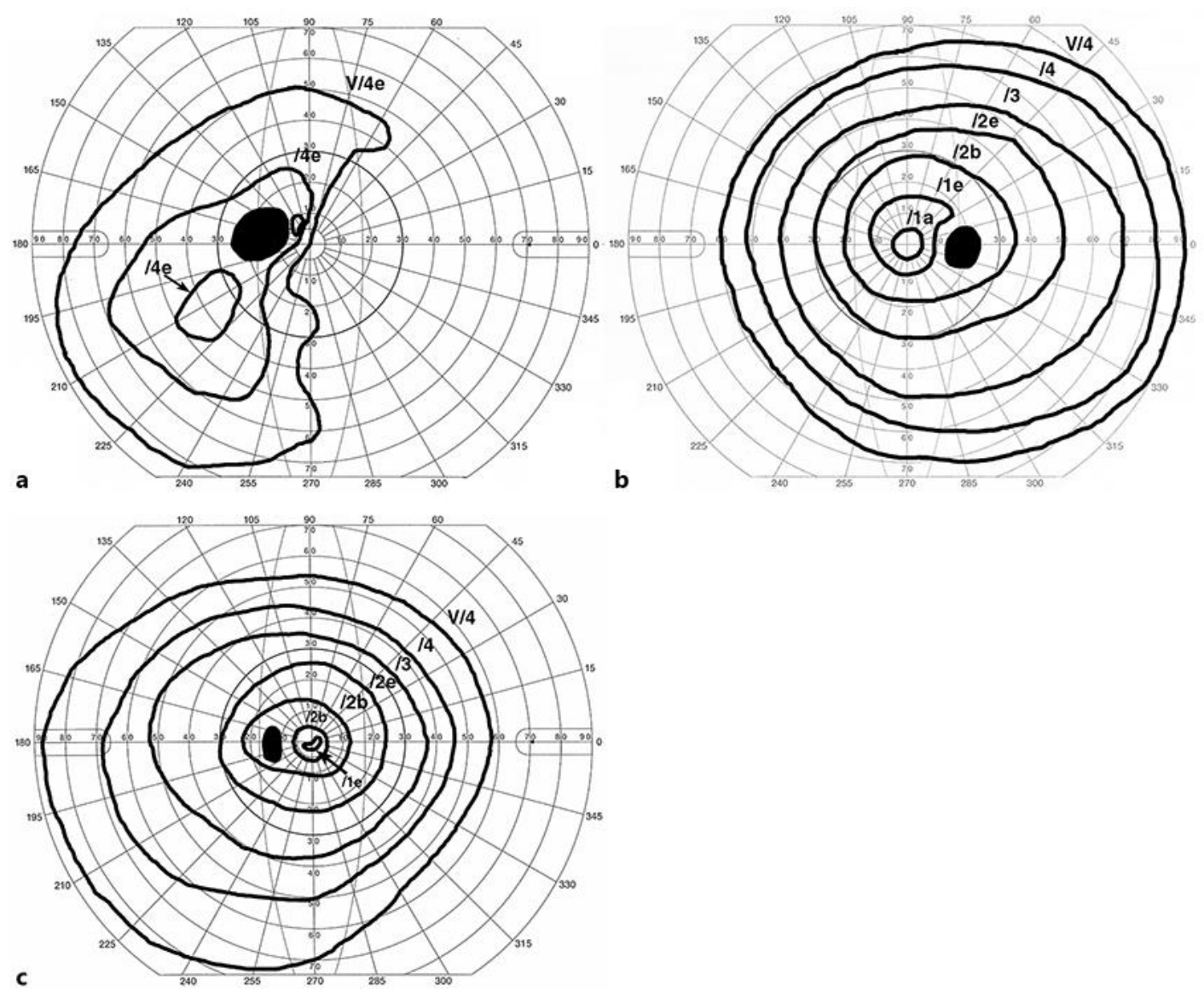

Fig. 1. Change in visual field before and after steroid pulse therapy. a The Goldmann kinetic visual field test demonstrating a defect in the nasal visual field and enlarged blind spot. $\mathbf{b}$ No visual defects were observed in the right eye. c After steroid pulse therapy, the visual field recovered completely. 


\section{Case Reports in Ophthalmology}
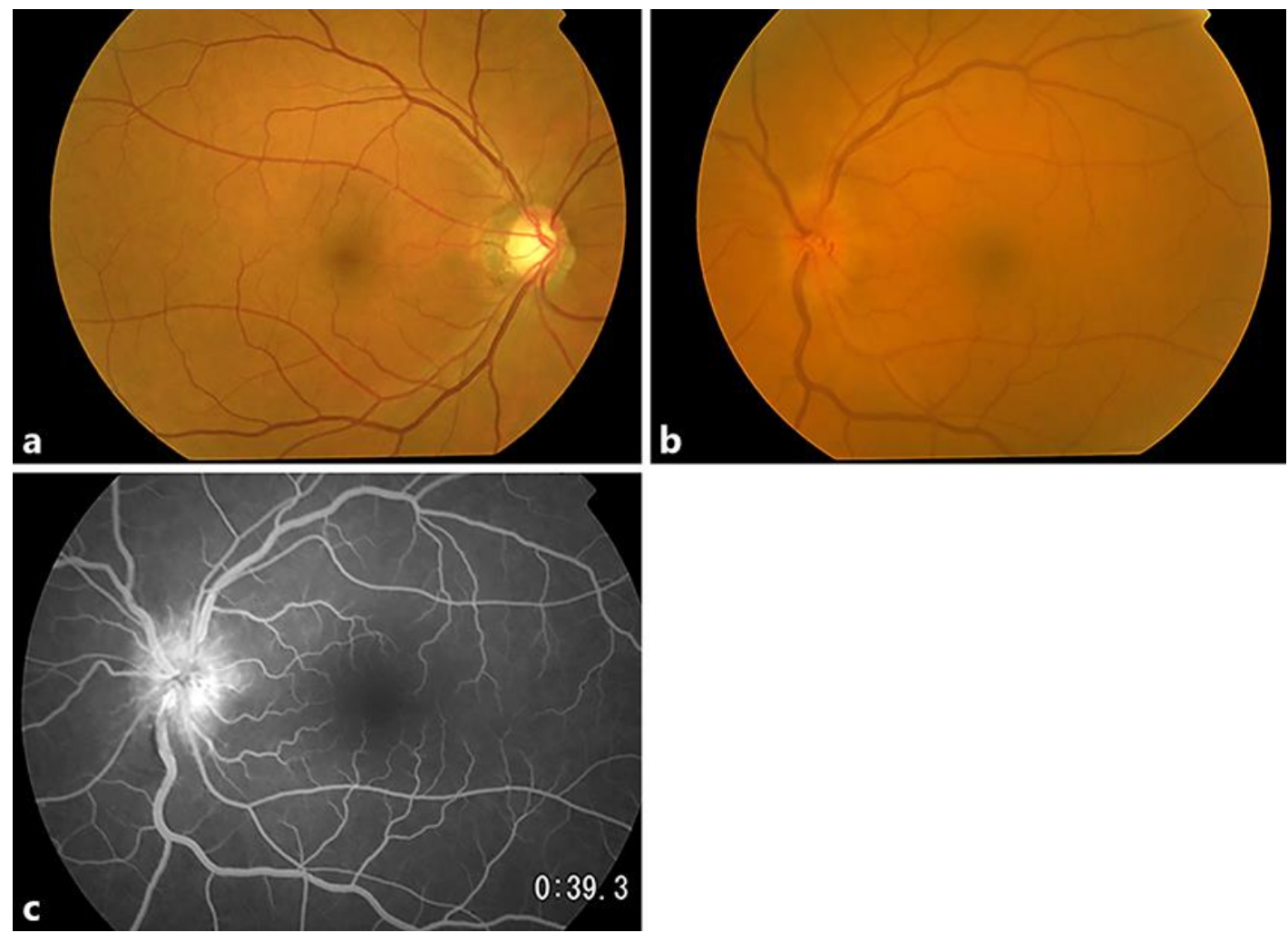

Fig. 2. Fundus photograph and fluorescein angiography of the optic disk. a A fundus photograph of the right eye showing large cupping of optic disk without visual field defect. $\mathbf{b}$ The optic disk of the left eye was swollen and retinal venous vessels demonstrated mild congestion without bleeding. c Fluorescent angiography showing optic disk leakage without a choroidal filling defect or other retinal abnormalities. The time after initiation was $39 \mathrm{~s}$. 


\section{Case Reports in Ophthalmology}

www.karger.com/cop
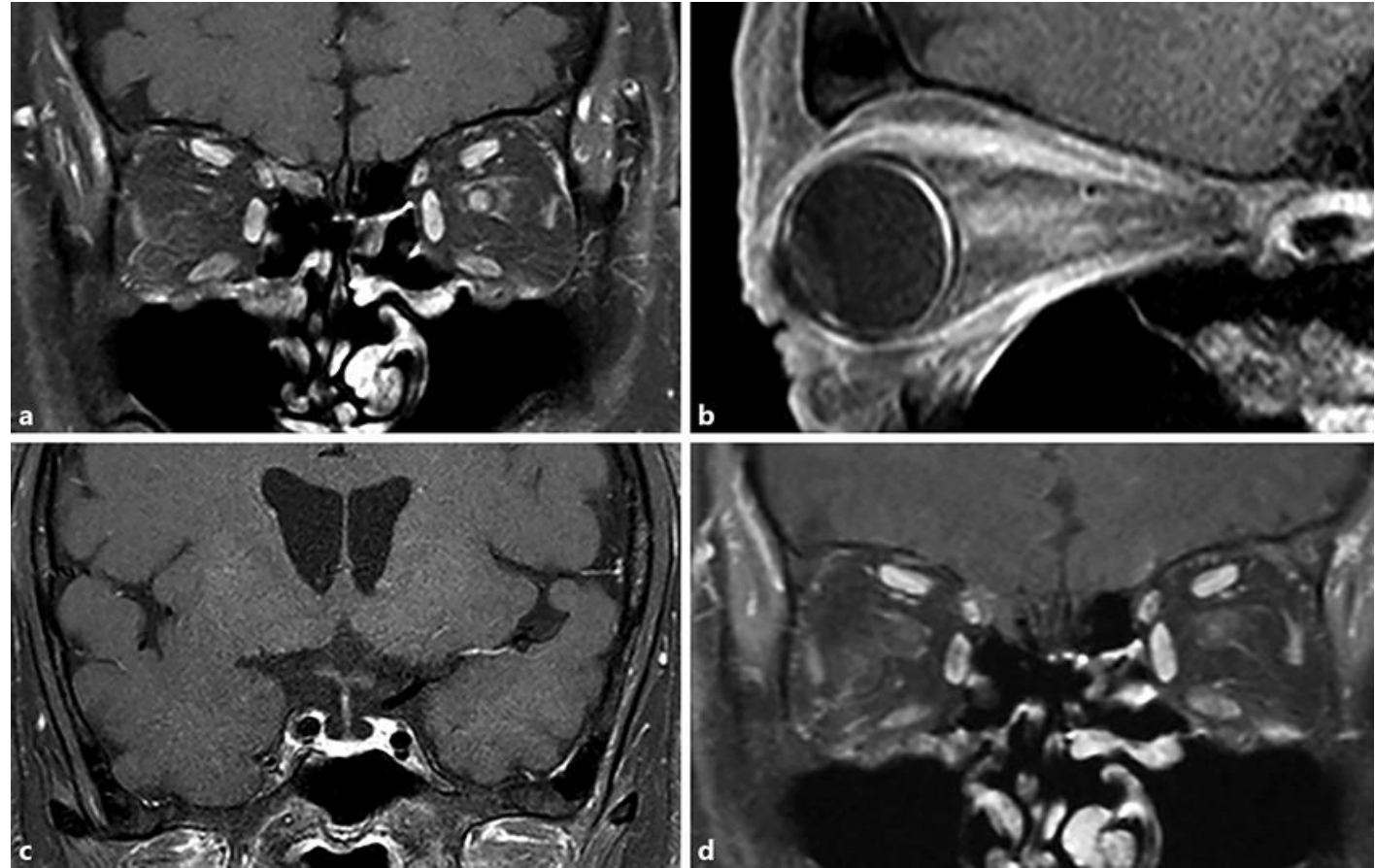

Fig. 3. Gadolinium-enhanced magnetic resonance imaging (MRI) before and after steroid pulse therapy. Gadolinium-enhanced MRI showed enhancement in the left optic neuritis on coronal (a) and sagittal (b) fat-suppressed T1-weighted imaging. c No abnormalities were detected in the pituitary body and stalks. d After steroid pulse therapy, the high-intensity area in the left optic nerve markedly decreased. 